\title{
Comparison of the efficacy of brolucizumab with natural disease progression in wet AMD using clinical data from the Phase III HAWK and HARRIER trials and modelled placebo data
}

Hansjürgen Agostini, Zufar Mulyukov, Miltiadis Tsilimbaris, Pilar Calvo, Felicitas Bucher, David Gaucher, Etienne Pigeolet, Valeria Colafrancesco \& Andreas Clemens

To cite this article: Hansjürgen Agostini, Zufar Mulyukov, Miltiadis Tsilimbaris, Pilar Calvo, Felicitas Bucher, David Gaucher, Etienne Pigeolet, Valeria Colafrancesco \& Andreas Clemens (2020): Comparison of the efficacy of brolucizumab with natural disease progression in wet AMD using clinical data from the Phase III HAWK and HARRIER trials and modelled placebo data, Current Eye Research, DOI: 10.1080/02713683.2020.1731832

To link to this article: https://doi.org/10.1080/02713683.2020.1731832

Accepted author version posted online: 17 Feb 2020.

Submit your article to this journal $\sqsubset$

山 Article views: 12

Q View related articles ¿

View Crossmark data $\nearrow$ 
Publisher: Taylor \& Francis

Journal: Current Eye Research

DOI: $10.1080 / 02713683.2020 .1731832$

Comparison of the efficacy of brolucizumab with natural disease progression in wet AMD using clinical data from the Phase III HAWK and HARRIER trials and modelled placebo data

Hansjürgen Agostini ${ }^{\mathrm{a}}$, Zufar Mulyukov ${ }^{\mathrm{b}}$, Miltiadis Tsilimbaris ${ }^{\mathrm{c}}$, Pilar Calvo ${ }^{\mathrm{d}}$, Felicitas Bucher ${ }^{\mathrm{a}}$, David Gaucher ${ }^{\mathrm{e}}$, Etienne Pigeolet ${ }^{\mathrm{b}}$, Valeria Colafrancesco ${ }^{\mathrm{f}}$, and Andreas Clemens ${ }^{\text {b, }}$ *

${ }^{a}$ Eye Center, Medical Center, Faculty of Medicine, University of Freiburg, Freiburg, Germany; ${ }^{b}$ Novartis Pharma AG, Basel, Switzerland; ${ }^{c}$ Department of Ophthalmology, University Hospital of Heraklion, Heraklion, Crete, Greece; ${ }^{d} I I S$-Aragon, Department of Ophthalmology, Miguel Servet University Hospital, Zaragoza, Spain; ${ }^{e}$ Nouvel Hôpital Civil, Hôpitaux Universitaires de Strasbourg, Strasbourg, France, ${ }^{f}$ Novartis Farmaceutica, SA, Barcelona, Spain; ${ }^{g}$ Department of Cardiology and Angiology I, Heart Center Freiburg University, Faculty of Medicine, University of Freiburg, Freiburg, Germany

*Corresponding author:

Dr Andreas Clemens, MD, PhD

Department of Ophthalmology, Regional Medical Director

Novartis Pharma AG, Postfach 4002

Basel, Switzerland

Telephone: +41616965836

Fax: +41613248001

Email: andreas.clemens@novartis.com

Target journal: Current Eye Research

Category: Short Communication 
Word limit: 1500 words; currently: 1499

Abstract limit: 300 words; currently: 206

Figure/table limit: 4; currently: 2

\section{Comparison of the efficacy of brolucizumab with natural disease progression in wet AMD using clinical data from the Phase III HAWK and HARRIER trials and modelled placebo data}

Aim: To compare the treatment effect of brolucizumab, a novel anti-vascular endothelial growth factor therapeutic, with a putative placebo in patients with wet age-related macular degeneration.

Materials and Methods: Clinical treatment-effect data from patients receiving brolucizumab $6 \mathrm{mg}$ in the HAWK and HARRIER studies were compared with modelled placebo data using a previously developed and validated indirect response, non-linear, mixed effects model describing the natural visual acuity decline in wet age-related macular degeneration. The placebo model incorporated patient-level data from the sham injection arms of the MARINA and PIER studies, corrected for baseline best corrected visual acuity and age difference between these studies and the HAWK and HARRIER studies.

Results: Compared with a modelled placebo, brolucizumab treatment was associated with an overall best corrected visual acuity gain of approximately 22 Early Treatment Diabetic Retinopathy Study letters at Week 48 and 28 letters at Week 96.

Conclusions: As anti-vascular endothelial growth factor therapy is now a standard of care for wet age-related macular degeneration, it is not feasible to conduct placebo-controlled trials for new wet age-related macular degeneration treatments. By allowing comparison with the natural decline in visual acuity without treatment, this analysis conveys the clinical importance of brolucizumab for the treatment of wet age-related macular degeneration.

Keywords: brolucizumab; wet age-related macular degeneration; natural disease course; putative placebo; indirect response model 


\section{Introduction}

Age-related macular degeneration (AMD) is a leading cause of vision loss globally, with a prevalence of $8.7 \%^{1,2}$. Although accounting for $\sim 10 \%$ of AMD cases, wet AMD (wAMD; also known as neovascular AMD) was responsible for $\sim 90 \%$ of AMD-associated vision loss before the advent of therapy in the $1980 \mathrm{~s}^{3,4}$. Initial thermal laser treatment was only effective in preventing wAMD-associated vision loss in a small number of cases, however, and often resulted in retinal scarring and recurrent neovascularisation ${ }^{5,6}$. From the late $1990 \mathrm{~s}$, photodynamic therapy was shown to slow progression of vision loss, although the majority of patients did not experience improvements in best corrected visual acuity (BCVA) ${ }^{7}$.

The introduction of anti-vascular endothelial growth factor (VEGF) therapy in wAMD substantially improved patient outcomes. Pivotal trials showed that intravitreal anti-VEGF therapy significantly improves BCVA, with $>16$ Early Treatment Diabetic Retinopathy Study (ETDRS) letters difference versus placebo after a year of treatment ${ }^{8,9}$. Following the approval of anti-VEGF therapies, such as ranibizumab, in the mid-2000s the rate of incident blindness and vision loss associated with wAMD reduced substantially ${ }^{10}$. Another anti-VEGF molecule, aflibercept, was subsequently approved for wAMD treatment in 2011 in the US and 2012 in the EU. With anti-VEGF therapy having become the gold standard for wAMD treatment, approval was based on demonstrating non-inferiority to ranibizumab in pivotal studies ${ }^{11}$.

On October $7^{\text {th }} 2019$, a new generation anti-VEGF molecule, brolucizumab, was approved by the FDA, and has also been submitted for marketing authorization to the EMA and licencing bodies in several other countries and regions. Brolucizumab has a high affinity for VEGF and will be the first humanized single-chain antibody fragment for ophthalmic use ${ }^{12}$. Its low molecular weight and size allows for more drug per dose and the potential for rapid and more effective tissue penetration compared with other anti-VEGF molecules ${ }^{12-14}$. Clinically, the efficacy of brolucizumab in wAMD was confirmed in the Phase III HAWK and HARRIER studies, which demonstrated non-inferiority of brolucizumab to aflibercept in BCVA while showing superior anatomical results; this was achieved with $>50 \%$ of patients on a 12 -weekly regimen directly after loading ${ }^{15}$.

As anti-VEGF therapy is now a standard of care in wAMD, a non-inferiority approach is necessary when evaluating new treatments and placebo-controlled studies are not considered feasible for ethical reasons. However, due to the lack of a placebo-controlled trial, the clinical value of this new anti-VEGF therapy relative to the natural disease course of wAMD may be underappreciated in the ophthalmology community. Therefore in this study, we have used a previously developed model of natural disease progression in wAMD to compare treatment effect data for brolucizumab with a putative placebo. 


\section{Materials and Methods}

\section{Clinical data}

In the Phase III HAWK and HARRIER trials, eligible patients were aged $\geq 50$ years and had untreated, active choroidal neovascular lesions secondary to AMD affecting the central subfield of the study eye as assessed by fluorescein angiography ${ }^{15}$. A BCVA between 78 and 23 letters, inclusive, in the study eye at screening and baseline using ETDRS testing were prerequisit for study inclusion, respectively. Patients received a brolucizumab injection at Weeks 0,4 , and 8 (loading phase) and thereafter every 12 weeks (q12w) but were interval adjusted to every 8 weeks $(\mathrm{q} 8 \mathrm{w})$ if disease activity was present ${ }^{15}$. In this study, patient-level data on the treatment effect of brolucizumab $6 \mathrm{mg}$ on BCVA from both HAWK and HARRIER through to Week 96 ${ }^{15,16}$ were compared with modelled data from untreated wAMD patients.

\section{Modelled data}

An empirical Bayesian indirect response, non-linear, mixed effects model of natural disease progression in wAMD and treatment effect in anti-VEGF treatment-naïve patients was previously developed using data from the ranibizumab Phase III ANCHOR, MARINA, PIER, and EXCITE studies ${ }^{8,9,17-19}$. Patient-level data from 298 patients from the sham arms of the MARINA ${ }^{9}$ and PIER ${ }^{18}$ studies were used to describe the natural decrease in BCVA with placebo. In this model, BCVA score was treated as non-stationary at baseline and decreased to steady state $\left(\mathrm{k}_{\text {in }} / \mathrm{k}_{\text {out }}\right)$ at the BCVA deterioration rate constant $\left(\mathrm{k}_{\text {out }}\right)$ without treatment ${ }^{17}$. BCVA at baseline influenced the rate of BCVA change over time and patient age influenced this rate through its correlation with baseline BCVA. No other covariates were identified that affected the BCVA time profile in sham treated patients ${ }^{17}$. The model was validated by comparing real outcomes with modelled outcomes using data from the HARBOR trial ${ }^{20}$, which was not used for model development ${ }^{17}$.

The putative placebo arm was simulated using the specific covariates of baseline BCVA and age of patients from the brolucizumab $6 \mathrm{mg}$ arms of the HAWK and HARRIER studies. The posterior distributions of the population parameters were used to sample the same number of subjects as the treated arms for both studies. Simulations were performed using RStan software version 2.17.3 (Stan Development Team, www.mc-stan.org).

\section{Results}

In the brolucizumab $6 \mathrm{mg}$ arms in the HAWK $(\mathrm{N}=360)$ and HARRIER ( $=370)$ studies, mean age at baseline was 76.7 and 74.8 years, respectively ${ }^{15}$. Mean baseline BCVA was 60.8 (HAWK) and 61.5 (HARRIER) ETDRS letters, and approximately $75 \%$ of study eyes had BCVA $\leq 70$ letters ${ }^{15}$. 
In our study, brolucizumab treatment was associated with a BCVA gain of $\sim 22$ ETDRS letters at Week 48 and 28 letters at Week 96 versus simulated sham injection for both the HAWK and HARRIER studies (Figure 1 and Table 1).

\section{Discussion}

Pivotal trials validated anti-VEGF-A therapy for wAMD with monthly treatment, showing greatly improved patient outcomes. However, the need for frequent clinic and injection visits coupled with the anticipated increased prevalence of patients with wAMD may lead to a clinical scenario that is difficult to sustain. The HAWK and HARRIER studies successfully evaluated an alternative treatment option, and demonstrated robust BCVA gains with brolucizumab dosed with a $\mathrm{q} 12 \mathrm{w}$ or $\mathrm{q} 8 \mathrm{w}$ regimen. As these trials had a non-inferiority design, it is difficult to assess the impact of this new therapeutic relative to natural disease progression in wAMD.

In this comparison with a putative placebo group, brolucizumab $6 \mathrm{mg}$ consistently showed BCVA gains, with approximately 28 ETDRS letters difference after 96 weeks of treatment for both the HAWK and HARRIER trials. This corresponds to the difference between mildmoderate visual impairment with near-normal reading ability and severe visual impairment with the requirement for high-power magnifiers and restricted field of vision ${ }^{21}$. In the absence of treatment, the same model describes a gradual decrease in BCVA across the patient population (although individual drops can be sudden) to a steady state of 11 ETDRS letters ${ }^{17}$ (the International Council of Ophthalmology defines near-blindness as $\leq 10$ ETDRS letters ${ }^{21}$ ). These data speak to the value of anti-VEGF therapy in wAMD.

It is not possible to directly compare treatment effect data for brolucizumab in the HAWK and HARRIER studies with placebo data from the MARINA and PIER studies due to differences in patient populations. Baseline BCVA in the sham arms of the MARINA and PIER studies was 67 ETDRS letters lower than in HAWK and HARRIER and patients were 2-3 years older ${ }^{9,15,18}$. Baseline BCVA, and to a lesser extent age, was shown to impact BCVA change over time under both treatment and placebo conditions. During model development it was determined that gender did not affect BCVA over time. Furthermore the status of choroidal neovascularization (CNV) type and baseline BCVA were confounded, and including CNV type into model did not improve it. Based on these aspects, differences in BCVA and age between studies were corrected for in the model used ${ }^{17}$. Furthermore, this model has been shown to hold true over the complete range of BCVA scores ${ }^{17}$.

The model provides a unique opportunity to demonstrate BCVA score behaviour under natural disease progression. Its accuracy in describing natural disease progression and treatment effect in anti-VEGF treatment-naïve patients has been shown through external validation using data from the HARBOR study. In that study, modelled data was shown to correlate well with actual data for ranibizumab, including prediction of a $2 \mathrm{mg}$ ranibizumab dose that was not used for model development ${ }^{17,20}$. An earlier version of the model, using 1-year data from the same studies, supported a label update approval by the EMA for individualised visual acuity-guided treatment with $0.5 \mathrm{mg}$ ranibizumab ${ }^{22}$. 
Limitations of the study include the relatively low number of placebo patients informing the model, totalling 298 patients from two studies ${ }^{17}$. In addition, unknown covariates that were not identified during model development, and therefore not used in simulations to adjust for differences in the HAWK and HARRIER populations, could influence the BCVA time profile in untreated patients.

Given the success of anti-VEGF therapies in wAMD, it is easy to forget the debilitating effects of this disease, where vision loss is almost guaranteed without treatment. In this study, brolucizumab provided vision gains of $\sim 22$ letters at Week 48 and $\sim 28$ letters at Week 96 versus natural (untreated) visual acuity decline in cohorts with mean baseline BCVA below 71 ETDRS letters. Furthermore, in HAWK and HARRIER, gains with brolucizumab treatment were achieved with $>50 \%$ of patients on a 12 -weekly retreatment regimen, commenced immediately after loading. Our analysis enables better understanding of the clinical value of this newgeneration intravitreal anti-VEGF treatment for wAMD. Future research will focus on understanding the utility of this new molecule in other anti-VEGF responsive indications.

\section{Acknowledgments}

Medical writing support was provided by Susan Browne, $\mathrm{PhD}$ of Novartis Ireland Ltd., in accordance with Good Publication Practice (GPP3) guidelines (http://www.ismpp.org/gpp3). The funding for this writing support was provided by Novartis.

\section{Conflicts of Interest}

Andreas Clemens and Etienne Pigeolet are employed by and hold shares in Novartis Pharma AG. Zufar Mulyukov is an employee of Novartis Pharma AG and Valeria Colafrancesco is an employee of Novartis Farmaceutica SA. David Gaucher is a board member for Novartis and Thea, and has received financial support for congress attendance from Bayer, FCI, and Allergan. Miltiadis Tsilimbaris has received research grants and financial support for congress attendance from Novartis, Bayer, Johnson \& Johnson, and Allergan. Pilar Calvo is a board member and has received lecture fees from Novartis, and financial support for congress attendance from Novartis, Thea, Bayer, and Allergan. Novartis has provided travel and study grants to Freiburg University Hospital, where Hansjürgen Agostini and Felicitas Bucher are employed. 


\section{References}

1. Schmidt-Erfurth U, Chong V, Loewenstein A, Larsen M, Souied E, Schlingemann R, Eldem B, Monés J, Richard G, Bandello F; European Society of Retina Specialists. Guidelines for the management of neovascular age-related macular degeneration by the European Society of Retina Specialists (EURETINA). Br J Ophthalmol. 2014;98(9):11441167.

2. Wong WL, Su X, Li X, Cheung CM, Klein R, Cheng CY, Wong TY. Global prevalence of age-related macular degeneration and disease burden projection for 2020 and 2040: a systematic review and meta-analysis. Lancet Glob Health. 2014;2(2):e106-16.

3. Yonekawa Y, Miller JW, Kim IK. Age-related macular degeneration: advances in management and diagnosis. J Clin Med. 2015;4(2):343-359.

4. Ferris FL, Fine SL, Hyman L. Age-related macular degeneration and blindness due to neovascular maculopathy. Arch Ophthalmol. 1984;102:1640-1642.

5. Ladas ID, Chatziralli IP, Kotsolis AI, Douvali M, Georgalas I, Theodossiadis PG, Rouvas AA. Intravitreal ranibizumab versus thermal laser photocoagulation in the treatment of extrafoveal classic choroidal neovascularization secondary to age-related macular degeneration. Ophthalmologica. 2012;228(2):93-101.

6. Kent C. Rev Ophthalmol. 2009. Available at: https://www.reviewofophthalmology.com/article/macular-degeneration-is-laser-still-relevant (accessed December 2019)

7. Bressler NM, Arnold J, Benchaboune M, Blumenkranz MS, Fish GE, Gragoudas ES, Lewis H, Schmidt-Erfurth U, Slakter JS, Bressler SB, et al. Verteporfin therapy of subfoveal choroidal neovascularization in patients with age-related macular degeneration: additional information regarding baseline lesion composition's impact on vision outcomes-TAP report No. 3. Arch Ophthalmol. 2002;120(11):1443-54. 
8. Brown DM, Kaiser PK, Michels M, Soubrane G, Heier JS, Kim RY, Sy JP, Schneider S; ANCHOR Study Group. Ranibizumab versus verteporfin for neovascular age-related macular degeneration. N Engl J Med. 2006;355(14):1432-1444.

9. Rosenfeld PJ, Brown DM, Heier JS, Boyer DS, Kaiser PK, Chung CY, Kim RY; MARINA Study Group. Ranibizumab for neovascular age-related macular degeneration. N Engl J Med. 2006;355(14):1419-1431.

10. Campbell JP, Bressler SB, Bressler NM. Impact of availability of anti-vascular endothelial growth factor therapy on visual impairment and blindness due to neovascular age-related macular degeneration. Arch Ophthalmol. 2012;130(6):794-5.

11. Heier JS, Brown DM, Chong V, Korobelnik JF, Kaiser PK, Nguyen QD, Kirchhof B, Ho A, Ogura Y, Yancopoulos GD, et al. Intravitreal aflibercept (VEGF trap-eye) in wet agerelated macular degeneration. Ophthalmology. 2012 Dec;119(12):2537-48.

12. Tietz J, Spohn G, Schmid G, Konrad J, Jampen S, Maurer P, Schmidt A, Escher D. Affinity and potency of RTH258 (ESBA1008), a novel inhibitor of vascular endothelial growth factor a for the treatment of retinal disorders. Invest Ophthalmol Vis Sci. 2015;56 [abstract 1501].

13. Escher D, Schmidt A, Steiner P, Maurer P, Weissgerber G. Single-chain antibody fragments in ophthalmology. Presented at the European Society of Retina Specialists $15^{\text {th }}$ EURETINA Congress; 2015 Sep 17-20; Nice, France.

14. Nimz EL, Van’t Land CW, Yáñez JA, Chastain JE. Intraocular and systemic pharmacokinetics of brolucizumab (RTH258) in nonhuman primates. Poster 4996 presented at the Annual Meeting of the Association for Research in Vision and Ophthalmology (ARVO); 2016 May 1-5; Seattle, USA.

15. Dugel PU, Koh A, Ogura Y, Jaffe GJ, Schmidt-Erfurth U, Brown DM, Gomes AV, Warburton J, Weichselberger A, Holz FG; HAWK and HARRIER Study Investigators. HAWK and HARRIER: Phase 3, multicenter, randomized, double-masked trials of 
brolucizumab for neovascular age-related macular degeneration. Ophthalmology. 2019;pii: S0161-6420(18)33018-5. doi: 10.1016/j.ophtha.2019.04.017. [Epub ahead of print].

16. Dugel PU, Jaffe G, Schmidt-Erfurth U, Ogura Y, Koh A, Weissgerber G, Weichselberger A, Holz, FG. Brolucizumab for neovascular AMD: the 2-Year HAWK and HARRIER results. Presented at the American Academy of Ophthalmology $122^{\text {nd }}$ Annual Meeting; 2018 Oct 27-30; Chicago, USA.

17. Mulyukov Z, Weber S, Pigeolet E, Clemens A, Lehr T, Racine A. Neovascular agerelated macular degeneration: a visual acuity model of natural disease progression and ranibizumab treatment effect. CPT Pharmacometrics Syst Pharmacol. 2018;7(10):660-669.

18. Regillo CD, Brown DM, Abraham P, Yue H, Ianchulev T, Schneider S, Shams N. Randomized, double-masked, sham-controlled trial of ranibizumab for neovascular agerelated macular degeneration: PIER Study year 1. Am J Ophthalmol. 2008;145(2):239-248.

19. Schmidt-Erfurth U, Eldem B, Guymer R, Korobelnik JF, Schlingemann RO, Axer-Siegel R, Wiedemann P, Simader C, Gekkieva M, Weichselberger A; EXCITE Study Group. Efficacy and safety of monthly versus quarterly ranibizumab treatment in neovascular agerelated macular degeneration: the EXCITE study. Ophthalmology. 2011;118(5):831-9.

20. Busbee BG, Ho AC, Brown DM, Heier JS, Suñer IJ, Li Z, Rubio RG, Lai P; HARBOR Study Group. Twelve-month efficacy and safety of $0.5 \mathrm{mg}$ or $2.0 \mathrm{mg}$ ranibizumab in patients with subfoveal neovascular age-related macular degeneration. Ophthalmology. 2013;120(5):1046-56.

21. International Council of Ophthalmology: visual standards aspects and ranges of vision loss with emphasis on population surveys.

http://www.icoph.org/downloads/visualstandardsreport.pdf (accessed December 2019)

22. Holz FG, Korobelnik JF, Lanzetta P, Mitchell P, Schmidt-Erfurth U, Wolf S, Markabi S, Schmidli H, Weichselberger A. The effects of a flexible visual acuity-driven ranibizumab treatment regimen in age-related macular degeneration: outcomes of a drug and disease model. Invest Ophthalmol Vis Sci. 2010;51(1):405-12. 
Table 1. Mean observed BCVA. BCVA = best corrected visual acuity; ETDRS = Early Treatment Diabetic Retinopathy Study; SE = standard error

\begin{tabular}{|l|c|c|c|c|c|}
\hline Study & Week & $\mathbf{n}$ & \multicolumn{3}{|c|}{ Mean observed BCVA, ETDRS letters (SE) } \\
\cline { 3 - 6 } & & & $\begin{array}{c}\text { Brolucizumab } \\
\mathbf{( 6 ~ m g )}\end{array}$ & $\begin{array}{c}\text { Simulated } \\
\text { sham }\end{array}$ & Delta \\
\hline HARRIER & 48 & 352 & $69.2(0.8)$ & $46.5(1.1)$ & $22.7(1.4)$ \\
\hline HAWK & 48 & 326 & $68.1(1.0)$ & $45.9(1.1)$ & $22.1(1.5)$ \\
\hline HARRIER & 96 & 342 & $68.7(0.9)$ & $41.1(1.3)$ & $27.6(1.5)$ \\
\hline HAWK & 96 & 304 & $68.1(1.0)$ & $40.6(1.2)$ & $27.5(1.6)$ \\
\hline
\end{tabular}


Figure 1. Mean BCVA in the HARRIER ( $n=352$ at 48 weeks and $n=342$ at 96 weeks) and HAWK ( $n=326$ at 48 weeks and $n=304$ at 96 weeks) studies versus putative placebo. In HAWK and HARRIER, patients received brolucizumab $3 \mathrm{mg} / 6 \mathrm{mg}$ at Weeks 0 , 4, and 8 (loading phase) and thereafter every 12 weeks but were interval adjusted to every 8 weeks if disease activity was present. Solid lines show brolucizumab $6 \mathrm{mg}$ treatment effect, dashed lines show modelled natural disease progression. Shaded areas show $95 \%$ confidence intervals. BCVA = best corrected visual acuity

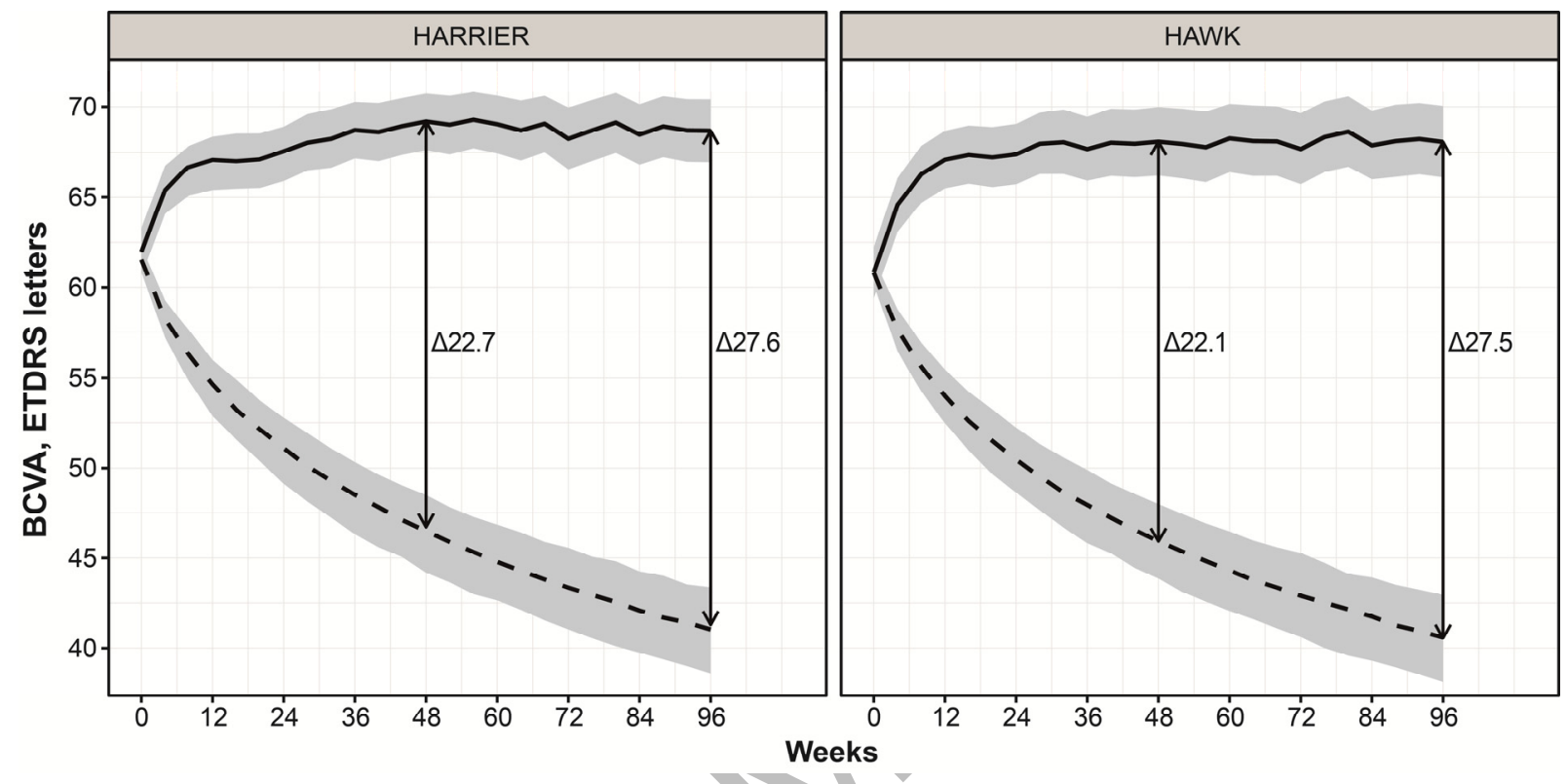

